\title{
AKT/mTOR as Novel Targets of Polyphenol Piceatannol Possibly Contributing to Inhibition of Proliferation of Cultured Prostate Cancer Cells
}

\author{
Tze-Chen Hsieh, ${ }^{1}$ Chia-Yi Lin, ${ }^{2}$ Hung-Yun Lin, ${ }^{3}$ and Joseph M. Wu ${ }^{1}$ \\ ${ }^{1}$ Department of Biochemistry \& Molecular Biology, New York Medical College, Valhalla, New York 10595, USA \\ ${ }^{2}$ Genome and Systems Biology Degree Program, National Taiwan University, Taipei 10617, Taiwan \\ ${ }^{3}$ Signal Transduction Laboratory, Ordway Research Institute, Albany, NY 12208, USA
}

Correspondence should be addressed to Tze-Chen Hsieh, tze-chen_hsieh@nymc.edu

Received 28 November 2011; Accepted 2 January 2012

Academic Editors: P.-L. Chang and T. Nelius

Copyright (C) 2012 Tze-Chen Hsieh et al. This is an open access article distributed under the Creative Commons Attribution License, which permits unrestricted use, distribution, and reproduction in any medium, provided the original work is properly cited.

The polyphenol piceatannol has shown inhibition against tyrosine and serine/threonine kinases. Whether piceatannol also exerts activity on the mammalian target of rapamycin (mTOR), a kinase involved in growth control of eukaryotic cells, is not known. In this study, we tested the effects of piceatannol on proliferation of androgen-dependent (AD) LNCaP and androgen-independent (AI) DU145 and PC-3 prostate cancer (CaP) cells. Suppression of AD and AI CaP cell growth by piceatannol was accompanied by cell cycle blockade in $\mathrm{G}_{1} / \mathrm{S}$ and $\mathrm{S}$ phases for $\mathrm{LNCaP}$ and PC-3 and induction of apoptosis in DU145 cells. Induction of apoptosis by piceatannol in DU145 cells was evident by reduced expression of poly(ADP-ribose) polymerase (PARP), cleavage of caspase 3 and apoptosis inducing factor AIF, and an increase in cytochrome c. The apoptotic changes occurred in concordance with DNA damage, supported by increased phosphorylated histone H2AX. Immunoblot analyses showed that exposure of different-stage $\mathrm{CaP}$ cells to piceatannol also resulted in cell-type-specific downregulation of mTOR and its upstream and downstream effector proteins, AKT and eIF-4E-BP1. We propose that the observed AKT and mTOR changes are new targets of piceatannol possibly contributing to its inhibitory activities on proliferation of CaP cells.

\section{Introduction}

Piceatannol $\left(3,3^{\prime}, 4,5^{\prime}\right.$-tetrahydroxy-trans-stilbene $)$ is a polyphenol found in food sources such as grapes, berries, peanuts, and sugar cane [1-4]. When first isolated from Euphorbia lagascae, piceatannol was found to exhibit antileukemic activity [5]. Subsequent experiments showed that piceatannol displays antioxidant $[6,7]$, anti-inflammatory $[8,9]$, and anticarcinogenic properties [10-12]. In cell model studies, piceatannol prevented TNF-induced $\mathrm{NF} \kappa \mathrm{B}$ activation $[9,13,14]$, by controlling the oxidoreductive status of cysteine-179 in IKK $\beta$ [4]. Suppression of lung metastasis occurred in Lewis-lung-carcinoma-bearing mice fed piceatannol-fortified diet [15].

Mechanistically, piceatannol has been investigated as an inhibitor for tyrosine kinases, including Syk [16-18], FAK $[19,20]$, and serine/threonine kinases [21]. Furthermore, piceatannol is also used to explore the role of the mitochondrial $\mathrm{F}_{0} \mathrm{~F}_{1}$-ATPase $[22,23]$, in relation to apoptosis. Recent studies show that piceatannol inhibits proliferation and induces cell cycle arrest and apoptosis in DU145 CaP cells [24-26] and that the anticellular effects of piceatannol are mediated by suppression of the cyclin-dependent protein kinase activities (CDKs) [26]. These results suggest that piceatannol might have chemopreventive potential for CaP.

The mTOR is a serine/threonine protein kinase that plays a crucial role in sensing the availability of nutrients for control of cell growth, generally conferring survival benefits $[27,28]$. Since mTOR is frequently deregulated in cancer and because, as mentioned, piceatannol acts as a potent kinase inhibitor, it is of interest to determine whether piceatannol might affect mTOR activity/expression and in turn disrupt mTOR-mediated signaling events. 
LNCaP

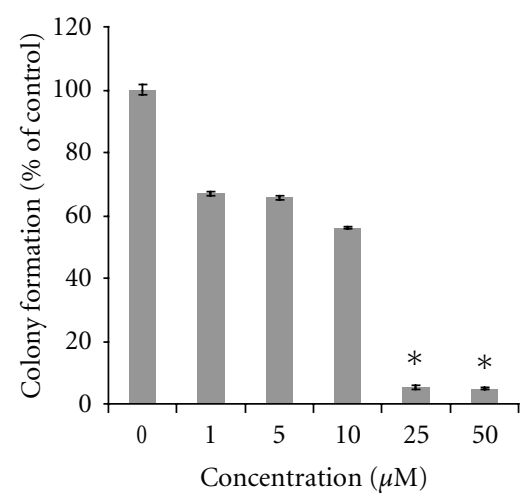

LNCaP

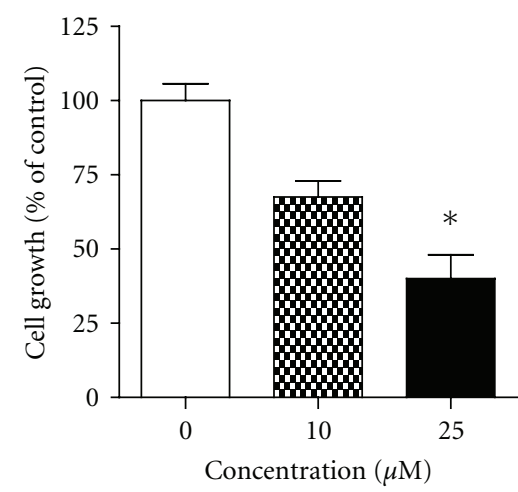

DU145

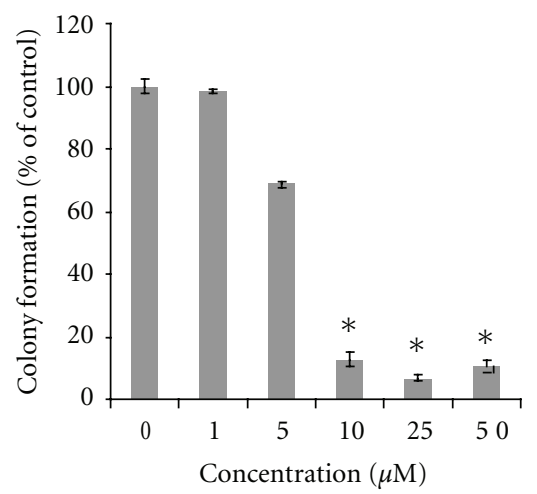

(a)

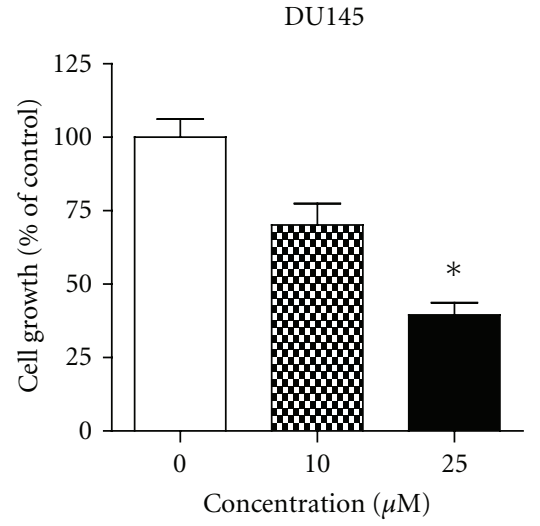

(b)

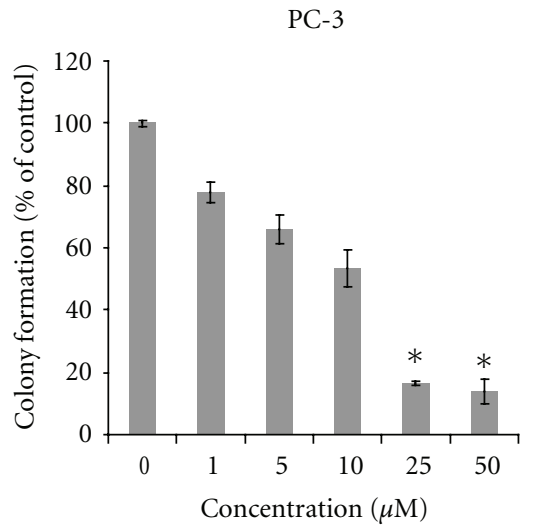

PC-3

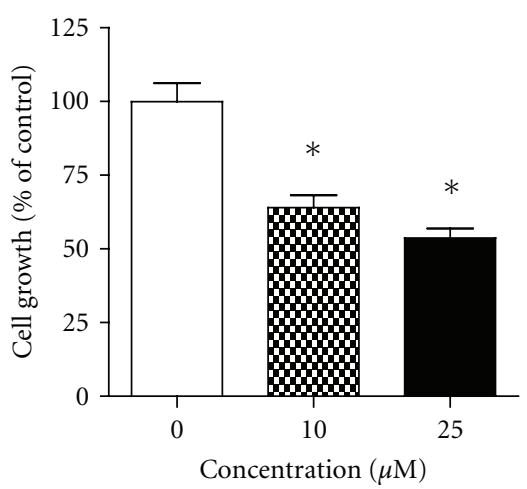

Figure 1: Effects of piceatannol on colony formation and cell proliferation in LNCaP, DU145, and PC-3 CaP cells. (a) Control of clonogenicity by piceatannol. Cells were plated in 6 -well tissue culture dishes at 2,000 cells $/ \mathrm{mL}$ and treated with increasing concentrations of piceatannol $(0,1,5,10,25$, and $50 \mu \mathrm{M})$. After 1 week, colonies were stained with $1.25 \%$ crystal violet and quantified by measuring the absorbance at $595 \mathrm{~nm}$. Dose-dependent suppression of colony formation by piceatannol was expressed as a percentage of control (set as $100 \%)$. Values are expressed as mean \pm SD for three separate experiments. The symbol represents statistical significance: ${ }^{*} P \leq 0.001$. (b) Control of cell growth by piceatannol. Cells were treated with increasing doses of piceatannol $(0,10$, and $25 \mu \mathrm{M})$. The cell numbers were determined at $72 \mathrm{~h}$ using a hemocytometer. The bars show dose-dependent suppression of growth, expressed as a percentage of control (set as $100 \%)$. Values are expressed as mean \pm SD for three experiments. The symbol represents statistical significance: ${ }^{*} P \leq 0.001$.

In this study, we tested the hypothesis that piceatannol controls proliferation of both androgen-dependent $(\mathrm{AD})$ and rogen-independent $(\mathrm{AI}) \mathrm{CaP}$ cells by targeting the expression of mTOR. We also determined whether piceatannol disrupts the mTOR signaling pathway in $\mathrm{CaP}$ cells by eliciting changes in mTOR and its upstream and downstream effector proteins: mTOR, protein kinase AKT, initiation factor eIF$4 \mathrm{E}$ regulatory binding protein eIF- $4 \mathrm{E}-\mathrm{BP} 1$, and ribosomal protein p70 S6 kinase. We found that piceatannol suppressed $\mathrm{AD}$ and $\mathrm{AI} \mathrm{CaP}$ cell proliferation and that its growth inhibitory activity was accompanied by reduced expression of mTOR and its key effectors AKT and eIF4EBP-1.

\section{Materials and Methods}

2.1. Reagents. Piceatannol was obtained from A.G. Scientific, Inc. (San Diego, CA). The translational control sample kit was from Cell Signaling Technology, Inc. (Beverly, MA). The primary antibodies for cyclins D1 and E, CDKs 2 and 6, AIF, caspase 3, cytochrome c, actin, and secondary antibodies were from Santa Cruz Biotechnology, Inc. (Santa Cruz, CA). The antibody against phosphorylated histone H2AX (Ser139) was from Upstate Biotechnology Inc. (Lake Placid, NY). The antibodies for PARP were from Biomol International, L.P. (Plymouth Meeting, PA). Fetal bovine serum (FBS), RPMI 1640, penicillin, and streptomycin were from Cellgro, Inc (Herndon, VA). All other chemicals and solvents used were of analytical grade.

2.2. Cell Culture, Colony Formation, and Proliferation Assays. Human LNCaP, DU145, and PC-3 cells were obtained from the American Tissue Culture Collection (Manassas, VA) and cultured in RPMI 1640 supplemented with penicillin, streptomycin, and 10\% heat-inactivated FBS, as described [29]. Piceatannol was dissolved in dimethyl sulfoxide (DMSO) and added to the culture media at the specified dose. Colony formation assay was performed as detailed [30]. Briefly, cells $(800-2000$ cells $/ \mathrm{mL}, 2 \mathrm{~mL} /$ well in 6-well plates) were 
incubated with increasing doses of piceatannol. Colonies were stained with $1.25 \%$ crystal violet, extracted with $10 \%$ acetic acid, and quantified by spectrometry at $595 \mathrm{~nm}$. The experiments were performed in triplicate. For cell proliferation assays, cells were seeded in 6-well plates at a density of $1 \times 10^{5}$ cells $/ \mathrm{mL}$ for LNCaP cells and $5 \times 10^{4}$ cells $/ \mathrm{mL}$ for DU145 and PC-3 cells. Following treatment, control and treated cells were assayed by trypan blue exclusion using a hemocytometer [29]. Harvested cells were washed with PBS and stored at $-80^{\circ} \mathrm{C}$ for subsequent biochemical analyses.

2.3. Cell Cycle Analysis. Cells were treated with 0, 10, and $25 \mu \mathrm{M}$ piceatannol for $72 \mathrm{~h}$, washed with PBS, and stained with $1.0 \mu \mathrm{g} / \mathrm{mL}$ DAPI (Sigma Chemical Co., St. Louis, MO). Cell cycle phase distribution was assayed by flow cytometry $[31,32]$. MultiCycle software program from Phoenix Flow Systems (San Diego, CA) was used to deconvolute the cellular DNA histograms and quantify the percentage of cells in the $\mathrm{G}_{1}, \mathrm{~S}$, and $\mathrm{G}_{2} \mathrm{M}$ phases. Induction of apoptosis was also assayed by flow cytometry, as the sub- $\mathrm{G}_{1}$ peak $[31,32]$.

2.4. Preparation of Whole-Cell Extracts and Western Blot Analysis. Cells were collected and lysed in ice-cold RIPA buffer, which contained $50 \mathrm{mM}$ Tris, $\mathrm{pH} 7.4,150 \mathrm{mM}$ $\mathrm{NaCl}, 1 \mathrm{mM}$ EDTA, 1\% Triton X-100, 1\% deoxycholate, $0.1 \%$ SDS, $1 \mathrm{mM}$ dithiothreitol, and $10 \mu \mathrm{L} / \mathrm{mL}$ protease inhibitor cocktail from Sigma-Aldrich Corp. (St. Louis, MO). Protein concentrations of cell lysates were determined by coomassie protein assay kit (Pierce, Rockford, IL) using bovine serum albumin BSA as the standard. For immunoblot analysis, lysates containing $10 \mu \mathrm{g}$ of protein were separated by $10 \%$ SDS-gel electrophoresis, followed by transfer to nitrocellulose membranes and blocked with TBST buffer ( $10 \mathrm{mM}$ Tris, pH 7.5, $100 \mathrm{mM} \mathrm{NaCl}$, and $0.05 \%$ Tween 20 ) containing 3\% nonfat dried milk. The blots were incubated with specific primary antibodies, followed by secondary antibodies. Immunoreactivity was detected by enhanced chemiluminescence (ECL) using the instructions provided by the manufacturer (Kirkegaard \& Perry Laboratories, Inc., Gaithersburg, MD). The expression of actin was used as loading control. The intensity of the specific immunoreactive bands was densitometrically quantified and expressed as a ratio relative to the expression of actin.

2.5. Data Analysis. The results were expressed as mean \pm standard deviation (SD). One-way ANOVA and Student's $t$ test were used to determine the significance of differences in measured variables between control and piceatannol-treated cells. Statistical significance was set at $P \leq 0.001$.

\section{Results}

3.1. Piceatannol-Inhibited Clonogenicity and Proliferation in $\mathrm{CaP}$ Cells. In previous studies by Kim and coworkers, DU145 cells maintained in DMEM/F12 culture media supplemented with $1 \%$ charcoal-treated FBS were followed by $24 \mathrm{~h}$ serum deprivation and then treatment by piceatannol; exposure to the polyphenol inhibited cell proliferation and induction of apoptosis [25]. It was of interest to ascertain whether piceatannol exerts a similar antiproliferative activity in $\mathrm{CaP}$ cells representing different stages of disease progression without prior serum depletion. Androgen-receptor(AR)-positive hormone-responsive LNCaP and AR-negative hormone-nonresponsive DU145 and PC-3 CaP cells were exposed to $0,1,5,10,25$, and $50 \mu \mathrm{M}$ piceatannol, and effects on colony formation were determined. The addition of $>10 \mu \mathrm{M}$ piceatannol caused $50 \%$ suppression in foci forming ability in all three cell lines tested (Figure 1(a)). Similarly, as assayed by trypan blue exclusion, a dose-dependent inhibition of proliferation was observed in the three cell lines treated for $72 \mathrm{~h}$ with 0,10 , and $25 \mu \mathrm{M}$ piceatannol (Figure 1(b)).

3.2. Piceatannol-Induced Cell Cycle Arrest and Apoptosis in $\mathrm{CaP}$ Cells. The nature of growth suppression by piceatannol was next studied by measuring effects on cell cycle distribution by flow cytometry. Exposure to piceatannol resulted in cell-type-dependent changes: (i) LNCaP cells showed $\mathrm{G}_{1} / \mathrm{S}$ arrest, evident by an increase in the $\mathrm{G}_{1}$ cell population and a corresponding diminution in S phase cells (Figure 2(a)). (ii) As previously noted $[25,26]$, a significant induction of apoptosis occurred in DU145 cells (Figure 2(a)), although, without altering cell cycle phase transition. (iii) $S$ phase accumulation, concomitant with reduction in percentage of $\mathrm{G}_{1}$ cells, was observed in PC-3 cells (Figure 2(a)).

The differential cell cycle effects elicited by piceatannol in LNCaP and PC-3 cells prompted us to assess the changes on the expression of cell cycle regulatory proteins by western blot analysis. As cyclins D1and E and CDK2/CDK6 play a pivotal role in controlling the cells entry from $\mathrm{G}_{1}$ into the $\mathrm{S}$ phase, the changes on their expression were first measured. In LNCaP cells, increased cyclin D1 but suppressed cyclin E expression with relative low to undetectable expression of CDK2 and CDK6 was found in piceatannol-exposed cells (Figure 2(b)), in partial support of the $G_{1}$ cell arrest (Figure 2(a)). For PC-3 cells, a dose-dependent reduction in cyclin D1/CDK6/CDK2 and induction of cyclin E expression were observed following treatment by piceatannol (Figure 2(b)), consistent with an increase in $S$ paralleled by a decrease in $\mathrm{G}_{1}$ phase transition (Figure $2(\mathrm{a})$ ).

To corroborate induction of apoptosis in piceatannolexposed DU145 cells (Figure 2(a)), the expression of several apoptosis-related genes was assayed. Levels of PARP and procaspase 3 were substantially decreased in cells treated for $72 \mathrm{~h}$ with 10 and $25 \mu \mathrm{M}$ piceatannol (Figure 2(c)). Other apoptosis marker changes induced by piceatannol included cleavage of matured $62-\mathrm{kD}$ AIF to its $57-\mathrm{kD}$ product [33] and increase in total cytochrome c (Figure 2(c)). Further analysis implicated DNA damage as a molecular antecedent for piceatannol-induced apoptosis as phosphorylation of Ser139 in histone H2AX, a marker for global DNA damage [34-36], showed a marked elevation in DU145 cells treated with 10 or $25 \mu \mathrm{M}$ piceatannol (Figure $2(\mathrm{~d})$ ).

3.3. Piceatannol-Suppressed $m T O R$ Expression, in Concordance with Reduced Levels of eIF4E-BP1 and AKT. To obtain 

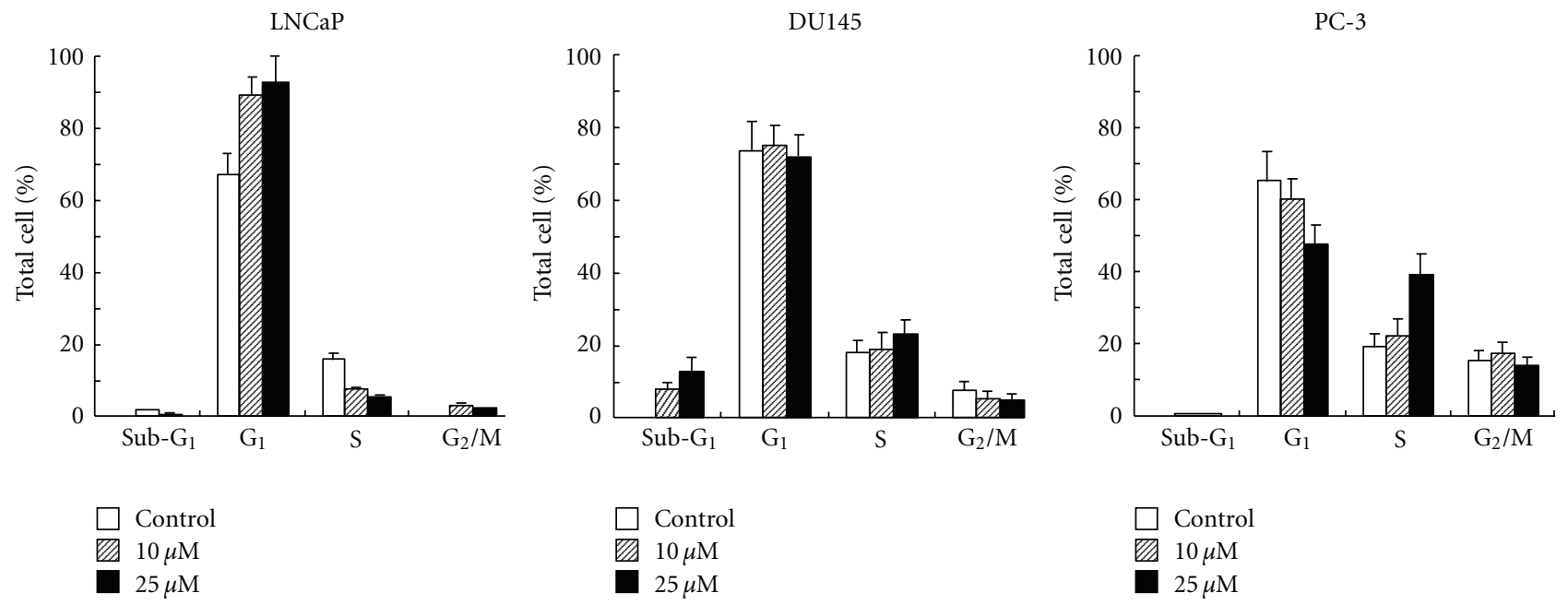

(a)
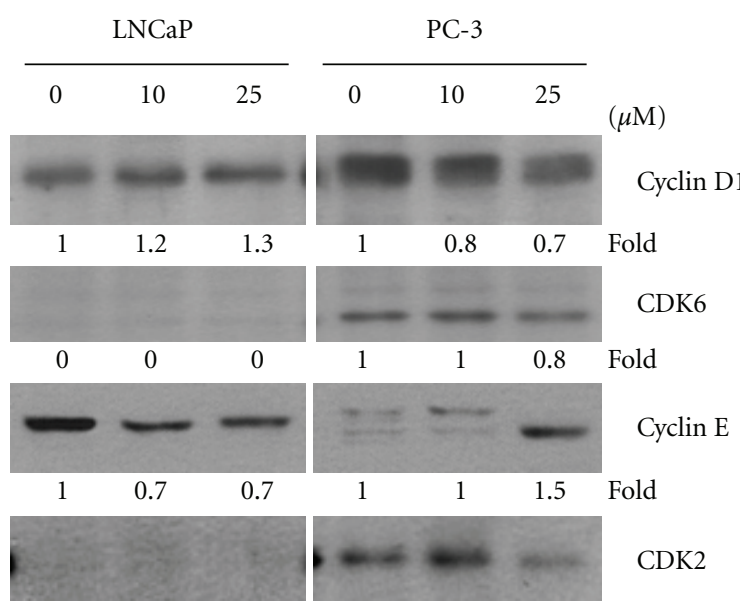

CDK2
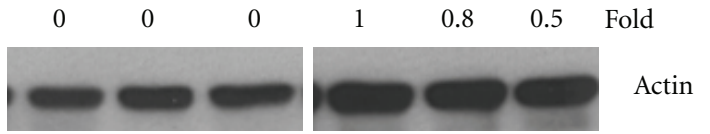

(b)
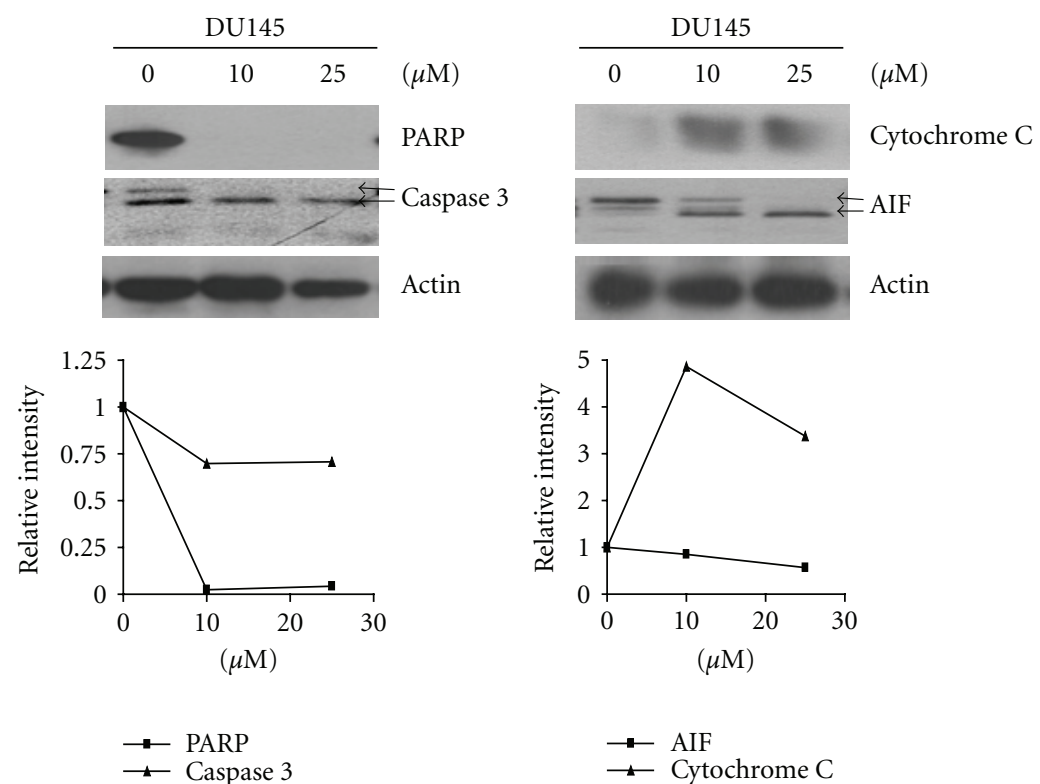

$:$ AIF
$\square$ Cytochrome C

(c)

Figure 2: Continued. 

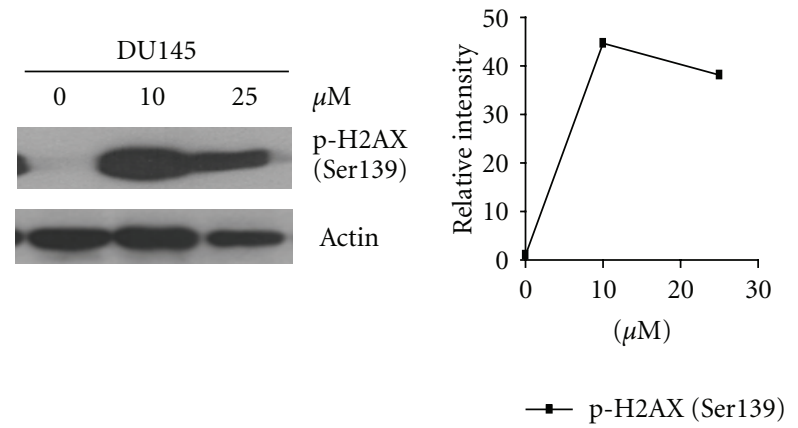

(d)

FIGURE 2: Effects of piceatannol on cell cycle control in LNCaP, DU145, and PC-3 CaP cells. Cells were treated with varying concentrations of piceatannol $(0,10$, and $25 \mu \mathrm{M})$ for $72 \mathrm{~h}$ and changes of cell cycle by piceatannol were further analyzed. (a) The effect on cell cycle distribution was analyzed by flow cytometry. The percentage of cells in $\mathrm{G}_{1}, \mathrm{~S}$, and $\mathrm{G}_{2} \mathrm{M}$ phases were calculated and values are expressed as mean $\pm \mathrm{SD}$. Cells with hypodiploid DNA content (sub- $\mathrm{G}_{1}$ ) representing fractions undergoing apoptosis were also calculated. (b) Changes on the expression of various cell cycle regulatory proteins by piceatannol in LNCaP and PC-3 cells. Western blot analysis of cyclins D1 E and CDK6/CDK2 protein expression levels in total cell lysate treated with piceatannol for $72 \mathrm{~h}$. (c) Effects of piceatannol treatment on apoptosis-associated proteins in DU145 cells. Changes in expression of PARP, caspase 3, cytochrome C, and AIF were further analyzed by western blot analysis. (d) Effects of piceatannol on DNA-damage-associated changes in DU145 cells were further analyzed by determining the changes in the expression of phosphorylated H2AX using western blot analysis. Actin expression was used as a loading control. The intensity of the specific immunoreactive bands was quantified by densitometry and expressed as a fold difference against actin.

further information on piceatannol-induced growth arrest, we tested the possible involvement of mTOR and mTORlinked signaling events. A dose-dependent reduction of mTOR expression occurred in all three $\mathrm{CaP}$ cells exposed to piceatannol, whereas differential expression of mTOR among three $\mathrm{CaP}$ cells was also observed (Figure 3(a)). Piceatannol also affected mTOR downstream effectors eIF$4 \mathrm{E}$ and eIF4E-BP1 [37, 38]. For example, a decrease in state of phosphorylation in Ser209 of eIF-4E and in Ser65 of eIF4EBP1 was observed in DU145 cells (Figure 3(b)), whereas reduced phosphorylated eIF4E (Ser209) was observed in LNCaP with undetectable changes on eIF4E-BP1 (Ser65), following treatment by $25 \mu \mathrm{M}$ piceatannol (Figure 3(b)). In PC-3 cells, piceatannol caused downregulation of phosphorylated eIF4E-BP1 (Ser65) while slightly increasing phosphorylated eIF4E (Ser209) (Figure 3(b)). We additionally tested if piceatannol affected $\mathrm{S} 6$ ribosomal protein levels and phosphorylation of p70-S6 kinase, both involved in control of cell proliferation, functioning as mTOR downstream targets $[39,40]$. All three CaP cell lines showed undetectable p70S6 kinase (Thr389) (data not shown). However, piceatannol inhibited p-S6 (Ser235/236) expression in LNCaP and PC3 cells (Figure 3(c)). Since AKT is an upstream modulator and activator of mTOR [41, 42], we analyzed changes in AKT expression. A dose-dependent suppression of total and Thr308-phosphorylated AKT was found in all three CaP cells treated by piceatannol (Figure 3(d)).

\section{Discussion}

We have investigated the growth suppressive activities of piceatannol by exposing cultured cells representing different stages of CaP, respectively, LNCaP (AD), DU145 and
PC-3 (both AI) to the polyphenol without prior serum depletion. A dose-dependent inhibition of proliferation and clonogenicity was observed in all $\mathrm{CaP}$ cells tested; PC3 cells were more effectively affected using the proliferation assay (Figure 1), while most significant reduction in colony formation was found in DU145 cells (Figure 1). These results confirmed and extended the reported growth control and apoptotic attributes of piceatannol $[25,26]$ and suggest that this polyphenol inhibits $\mathrm{CaP}$ proliferation irrespective of cellular dependency on hormones or culture conditions.

Previously, it has been reported that piceatannol exerts selectivity and potency against DU145 CaP cells [25] compared to normal prostate PWR-1E cells $[25,26]$ and that it induces cell cycle arrest and apoptosis via the inhibition of CDK activity and activation of the death receptor/mitochondrial-dependent pathways, respectively $[25,26]$. To more fully glean its anti-CaP potential, we sought to elucidate further insights into its mechanism of action. In DU145 cell studies we have found that exposure to piceatannol resulted in (i) PARP and caspase 3 changes (Figure 2(c)) implicating the involvement of caspase-dependent mode of cell death, (ii) AIF and cytochrome c changes (Figure 2(c)) implicating the involvement of mitochondria-dependent, caspase-independent mode of cell death, (iii) increased histone H2AX Ser139 phosphorylation (Figure 2(d)) suggesting coupling between induction of apoptosis with piceatannol-elicited DNA damage, and (iv) disruption of mTOR signaling, as evident by downregulation of AKT, mTOR, and eIF-4E-BP1 protein expression. Furthermore, suppression of proliferation and differential downregulation of AKT/mTOR expression were also found in LNCaP and PC-3 cells treated with piceatannol. The demonstration that 


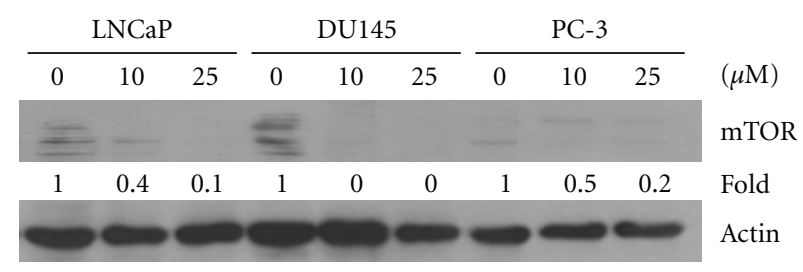

(a)

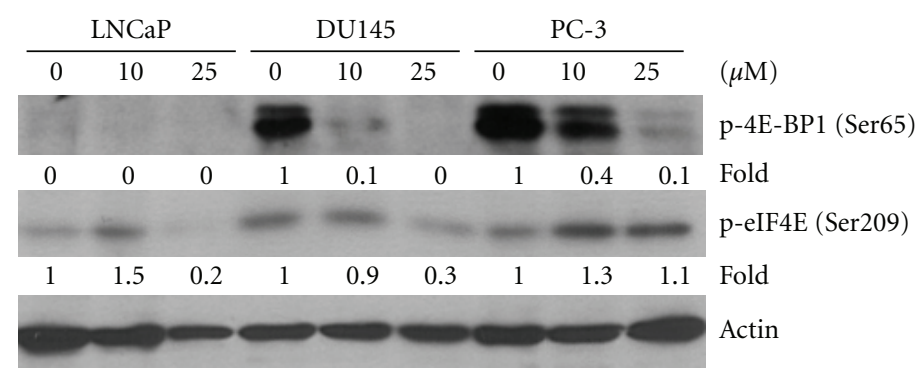

(b)

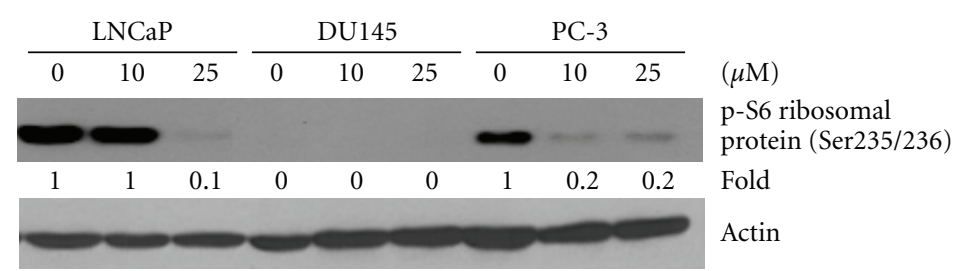

(c)

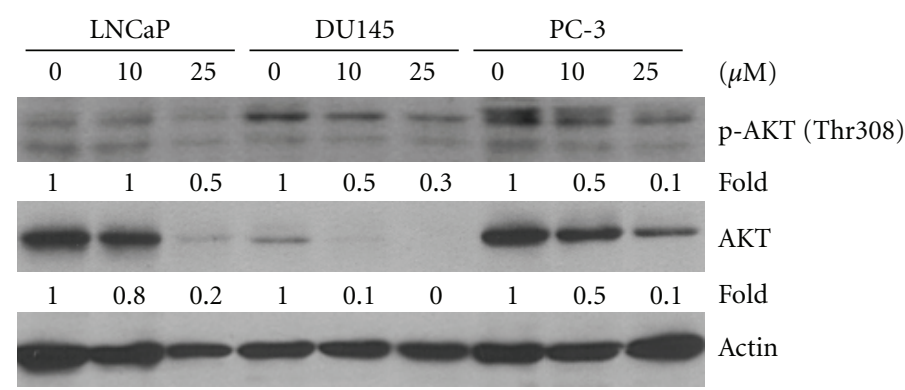

(d)

FIGURE 3: Effects of exposure to piceatannol on mTOR and its downstream p-4E-BP1/p-eIF4E and upstream AKT expression in LNCaP, DU145, and PC-3 cells. Cells were treated with varying concentrations of piceatannol $(0,10$, and $25 \mu \mathrm{M})$ for $72 \mathrm{~h}$, and immunoblot analysis was used to assess the changes in protein levels of (a) mTOR, (b) phosphorylated p-4E-BP1 (Ser65), and p-eIF4E (Ser209) (c) phosphorylated p-S6 ribosomal protein (Ser235/236), (d) total and phosphorylated AKT (Thr308). In each case, actin was used as a loading control. The intensity of the specific immunoreactive bands was densitometrically quantified and expressed as a fold difference against actin.

piceatannol induces marked reduction in AKT and mTOR levels in CaP cells (Figure 3 ) is significant and suggests a novel mechanism by which this polyphenol acts as a dietary agent for chemoprevention of CaP. Conceivably, piceatannol may affect $\mathrm{CaP}$ cell proliferation by targeting protein synthesis, mediated in part through the AKT/mTOR/eIF-4E-BP1 pathway. AKT is robustly activated in various cancers and the AKT signaling cascade is well integrated with growthfactor-mediated pathways in $\mathrm{CaP}$, having implications in $\mathrm{CaP}$ survival and development $[41,43]$. There is a wealth of evidence in support of the profound role mTOR plays in CaP. For example, inhibitors of the mTOR pathway have been shown to restrict $\mathrm{CaP}$ cell proliferation. Furthermore, suppression of mTOR by rapamycin effectively reverses AKTdependent prostatic intraepithelial neoplasia (PIN) in mouse model studies [43].

Since mTOR also acts as a molecular sensor and enforcer of cellular responses to changes in growth factors and nutrient status of the cell, by turning on the synthesis of proteins including those critically involved in the control of growth and cell cycle phase transition $[44,45]$, we assayed changes in one of the downstream translational effectors of mTOR, eIF-4E, and eIF-4E-BP1 known to be involved in mRNA assembly into productive initiation complexes in eukaryotic protein synthesis. Both were found to be modulated by piceatannol, suggesting that this dietary agent acts by 
Proposed mechanism of action of piceatannol

(a) Induction of apoptosis

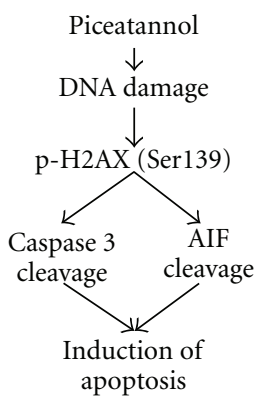

(b) Control of cell proliferation

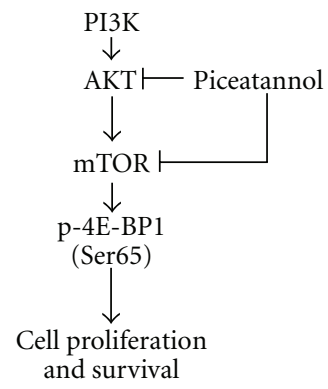

Figure 4: A model mechanism on the ability of piceatannol to induce apoptosis and suppress cell proliferation in $\mathrm{CaP}$ cells is proposed. (a) For induction of apoptosis, the effects of piceatannol is postulated to occur secondary to DNA damage accompanied by increased phosphorylation of H2AX at Ser139, which facilitate and culminate in the cleavage of caspase 3 and AIF. (b) for suppression of proliferation, piceatannol is proposed to inhibit the AKT/mTOR signaling pathway resulting in suppression of $\mathrm{CaP}$ cell proliferation and survival.

regulating the dynamics of interplay between the engagement and sequestration of initiation factors with a dominant role in mRNA binding and translation and in the regulation of specific mRNA recruitment from mRNA-ribonucleoprotein particles for participation in protein synthesis and growth control. One aspect of our current hypothesis is that the mTOR signaling pathway is a potential target of piceatannol and that $\mathrm{CaP}$ showing propensity for activation by mTOR might be selectively responsive to treatment by piceatannol. Figure 4 depicts the proposed anti-CaP mechanism(s) of piceatannol.

\section{Conflict of Interests}

The authors declare no conflict of interests.

\section{Acknowledgment}

This paper was supported in part by the Intramural Sponsored Research Program of New York Medical College to T.C. Hsieh.

\section{References}

[1] E. Cantos, J. C. Espin, M. J. Fernandez, J. Oliva, and F. A. Tomas-Barberan, "Postharvest UV-C-irradiated grapes as a potential source for producing stilbene-enriched red wines," Journal of Agricultural and Food Chemistry, vol. 51, no. 5, pp. 1208-1214, 2003.

[2] A. M. Rimando, W. Kalt, J. B. Magee, J. Dewey, and J. R. Ballington, "Resveratrol, pterostilbene, and piceatannol in vaccinium berries," Journal of Agricultural and Food Chemistry, vol. 52, no. 15, pp. 4713-4719, 2004.

[3] K. L. Ku, P. S. Chang, Y. C. Cheng, and C. Y. Lien, "Production of stilbenoids from the callus of arachis hypogaea: a novel source of the anticancer compound piceatannol," Journal of Agricultural and Food Chemistry, vol. 53, no. 10, pp. 38773881, 2005.

[4] P. S. Son, S. A. Park, H. K. Na, D. M. Jue, S. Kim, and Y. J. Surh, "Piceatannol, a catechol-type polyphenol, inhibits phorbol ester-induced NF- $\kappa$ B activation and cyclooxygenase2 expression in human breast epithelial cells: cysteine 179 of IKK $\beta$ as a potential target," Carcinogenesis, vol. 31, no. 8, pp. 1442-1449, 2010.

[5] N. R. Ferrigni, J. L. McLaughlin, R. G. Powell, and C. R. Smith Jr., "Use of potato disc and brine shrimp bioassays to detect activity and isolate piceatannol as the antileukemic principle from the seeds of Euphorbia lagascae," Journal of Natural Products, vol. 47, no. 2, pp. 347-352, 1984.

[6] T. Yokozawa and Y. J. Kim, "Piceatannol inhibits melanogenesis by its antioxidative actions," Biological and Pharmaceutical Bulletin, vol. 30, no. 11, pp. 2007-2011, 2007.

[7] M. Rossi, F. Caruso, C. Opazo, and J. Salciccioli, "Crystal and molecular structure of piceatannol; scavenging features of resveratrol and piceatannol on hydroxyl and peroxyl radicals and docking with transthyretin," Journal of Agricultural and Food Chemistry, vol. 56, no. 22, pp. 10557-10566, 2008.

[8] B. Djoko, R. Y. Chiou, J. J. Shee, and Y. W. Liu, "Characterization of immunological activities of peanut stilbenoids, arachidin-1, piceatannol, and resveratrol on lipopolysaccharide-induced inflammation of RAW 264.7 macrophages," Journal of Agricultural and Food Chemistry, vol. 55, no. 6, pp. 2376-2383, 2007.

[9] D. Liu, D. H. Kim, J. M. Park, H. K. Na, and Y. J. Surh, "Piceatannol inhibits phorbol ester-induced NF- $\kappa$ B activation and COX-2 expression in cultured human mammary epithelial cells," Nutrition and Cancer, vol. 61, no. 6, pp. 855-863, 2009.

[10] T. Wieder, A. Prokop, B. Bagci et al., "Piceatannol, a hydroxylated analog of the chemopreventive agent resveratrol, is a potent inducer of apoptosis in the lymphoma cell line BJAB and in primary, leukemic lymphoblasts," Leukemia, vol. 15, no. 11 , pp. 1735-1742, 2001.

[11] F. Wolter, A. Clausnitzer, B. Akoglu, and J. Stein, "Piceatannol, a natural analog of resveratrol, inhibits progression through the S phase of the cell cycle in colorectal cancer cell lines," Journal of Nutrition, vol. 132, no. 2, pp. 298-302, 2002.

[12] M. Larrosa, F. A. Tomás-Barberán, and J. C. Espín, “The grape and wine polyphenol piceatannol is a potent inducer of apoptosis in human SK-Mel-28 melanoma cells," European Journal of Nutrition, vol. 43, no. 5, pp. 275-284, 2004.

[13] K. Ashikawa, S. Majumdar, S. Banerjee, A. C. Bharti, S. Shishodia, and B. B. Aggarwal, "Piceatannol inhibits TNFinduced NF- $\kappa \mathrm{B}$ activation and NF- $\kappa \mathrm{B}$-mediated gene expression through suppression of I $\kappa$ Balpha kinase and $\mathrm{p} 65$ phosphorylation," Journal of Immunology, vol. 169, no. 11, pp. 6490-6497, 2002.

[14] J. Youn, J. S. Lee, H. K. Na, J. K. Kundu, and Y. J. Surh, "Resveratrol and piceatannol inhibit iNOS expression and NF- $\kappa$ B activation in dextran sulfate sodium-induced mouse colitis," Nutrition and Cancer, vol. 61, no. 6, pp. 847-854, 2009.

[15] Y. Kimura, K. Baba, and H. Okuda, "Inhibitory effects of active substances isolated from Cassia garrettiana heartwood on tumor growth and lung metastasis in Lewis lung carcinomabearing mice-part 2," Anticancer Research, vol. 20, no. 5, pp. 2923-2930, 2000.

[16] J. M. Oliver, D. L. Burg, B. S. Wilson, J. L. McLaughlin, and R. L. Geahlen, "Inhibition of mast cell Fc epsilon R1-mediated signaling and effector function by the Syk-selective inhibitor, 
piceatannol," Journal of Biological Chemistry, vol. 269, no. 47, pp. 29697-29703, 1994.

[17] H. E. Speich, S. Grgurevich, T. J. Kueter, A. D. Earhart, S. M. Slack, and L. K. Jennings, "Platelets undergo phosphorylation of Syk at Y525/526 and Y352 in response to pathophysiological shear stress," American Journal of Physiology, vol. 295, no. 4, pp. C1045-C1054, 2008.

[18] Y. Takada and B. B. Aggarwal, "TNF activates Syk protein tyrosine kinase leading to TNF-induced MAPK activation, NF- $\kappa$ B activation, and apoptosis," Journal of Immunology, vol. 173, no. 2, pp. 1066-1077, 2004.

[19] R. L. Geahlen and J. L. McLaughlin, "Piceatannol (3,4,3',5'tetrahydroxy-trans-stilbene) is a naturally occurring proteintyrosine kinase inhibitor," Biochemical and Biophysical Research Communications, vol. 165, no. 1, pp. 241-245, 1989.

[20] D. A. Law, L. Nannizzi-Alaimo, K. Ministri et al., "Genetic and pharmacological analyses of Syk function in alphaIIb $\beta 3$ signaling in platelets," Blood, vol. 93, no. 8, pp. 2645-2652, 1999.

[21] B. H. Wang, Z. X. Lu, and G. M. Polya, "Inhibition of eukaryote serine/threonine-specific protein kinases by piceatannol," Planta Medica, vol. 64, no. 3, pp. 195-199, 1998.

[22] J. Zheng and V. D. Ramirez, "Piceatannol, a stilbene phytochemical, inhibits mitochondrial F0F1-ATPase activity by targeting the F1 complex," Biochemical and Biophysical Research Communications, vol. 261, no. 2, pp. 499-503, 1999.

[23] J. R. Gledhill, M. G. Montgomery, A. G. Leslie, and J. E. Walker, "Mechanism of inhibition of bovine F1-ATPase by resveratrol and related polyphenols," Proceedings of the National Academy of Sciences of the United States of America, vol. 104, no. 34, pp. 13632-13637, 2007.

[24] B. E. Barton, J. G. Karras, T. F. Murphy, A. Barton, and H. F. Huang, "Signal transducer and activator of transcription 3 (STAT3) activation in prostate cancer: direct STAT3 inhibition induces apoptosis in prostate cancer lines," Molecular Cancer Therapeutics, vol. 3, no. 1, pp. 11-20, 2004.

[25] E. J. Kim, H. Park, S. Y. Park, J. G. Jun, and J. H. Park, “The grape component piceatannol induces apoptosis in DU145 human prostate cancer cells via the activation of extrinsic and intrinsic pathways," Journal of Medicinal Food, vol. 12, no. 5, pp. 943-951, 2009.

[26] Y. M. Lee, D. Y. Lim, H. J. Cho et al., "Piceatannol, a natural stilbene from grapes, induces G1 cell cycle arrest in androgen-insensitive DU145 human prostate cancer cells via the inhibition of CDK activity," Cancer Letters, vol. 285, no. 2, pp. 166-173, 2009.

[27] L. Kopelovich, J. R. Fay, C. C. Sigman, and J. A. Crowell, "The mammalian target of rapamycin pathway as a potential target for cancer chemoprevention," Cancer Epidemiology Biomarkers and Prevention, vol. 16, no. 7, pp. 1330-1340, 2007.

[28] L. Ciuffreda, C. D. Sanza, U. C. Incani, and M. Milella, "The mTOR pathway: a new target in cancer therapy," Current Cancer Drug Targets, vol. 10, no. 5, pp. 484-495, 2010.

[29] T. C. Hsieh and J. M. Wu, "Differential effects on growth, cell cycle arrest, and induction of apoptosis by resveratrol in human prostate cancer cell lines," Experimental Cell Research, vol. 249, no. 1, pp. 109-115, 1999.

[30] S. Elangovan, T. C. Hsieh, and J. M. Wu, "Growth inhibition of human MDA-MB-231 breast cancer cells by delta-tocotrienol is associated with loss of cyclin D1/CDK4 expression and accompanying changes in the state of phosphorylation of the retinoblastoma tumor suppressor gene product," Anticancer Research, vol. 28, no. 5, pp. 2641-2647, 2008.
[31] A. M. DiPietrantonio, T. C. Hsieh, S. C. Olson, and M. W. U. Joseph, "Regulation of G1/S transition and induction of apoptosis in HL-60 leukemia cells by fenretinide (4HPR)," International Journal of Cancer, vol. 78, no. 1, pp. 53-61, 1998.

[32] Z. Darzynkiewicz, E. Bedner, and P. Smolewski, "Flow cytometry in analysis of cell cycle and apoptosis," Seminars in Hematology, vol. 38, no. 2, pp. 179-193, 2001.

[33] H. Otera, S. Ohsakaya, Z. Nagaura, N. Ishihara, and K. Mihara, "Export of mitochondrial AIF in response to proapoptotic stimuli depends on processing at the intermembrane space," EMBO Journal, vol. 24, no. 7, pp. 1375-1386, 2005.

[34] E. P. Rogakou, D. R. Pilch, A. H. Orr, V. S. Ivanova, and W. M. Bonner, "DNA double-stranded breaks induce histone H2AX phosphorylation on serine 139," Journal of Biological Chemistry, vol. 273, no. 10, pp. 5858-5868, 1998.

[35] J. P. Banath and P. L. Olive, "Expression of phosphorylated histone $\mathrm{H} 2 \mathrm{AX}$ as a surrogate of cell killing by drugs that create DNA double-strand breaks," Cancer Research, vol. 63, no. 15, pp. 4347-4350, 2003.

[36] T. Tanaka, H. D. Halicka, X. Huang, F. Traganos, and Z. Darzynkiewicz, "Constitutive histone H2AX phosphorylation and ATM activation, the reporters of DNA damage by endogenous oxidants," Cell Cycle, vol. 5, no. 17, pp. 19401945, 2006.

[37] L. Furic, L. Rong, O. Larsson et al., "EIF4E phosphorylation promotes tumorigenesis and is associated with prostate cancer progression," Proceedings of the National Academy of Sciences of the United States of America, vol. 107, no. 32, pp. 14134-14139, 2010.

[38] D. Yu, C. Scott, W. W. Jia et al., "Targeting and killing of prostate cancer cells using lentiviral constructs containing a sequence recognized by translation factor eIF4E and a prostate-specific promoter," Cancer Gene Therapy, vol. 13, no. 1, pp. 32-43, 2006.

[39] N. Pullen and G. Thomas, "The modular phosphorylation and activation of p70(s6k)," FEBS Letters, vol. 410, no. 1, pp. 7882, 1997.

[40] A. Dufner and G. Thomas, "Ribosomal S6 kinase signaling and the control of translation," Experimental Cell Research, vol. 253, no. 1, pp. 100-109, 1999.

[41] J. R. Graff, B. W. Konicek, A. M. McNulty et al., "Increased AKT activity contributes to prostate cancer progression by dramatically accelerating prostate tumor growth and diminishing p27(Kip1) expression," Journal of Biological Chemistry, vol. 275, no. 32, pp. 24500-24505, 2000.

[42] C. W. Kinkade, M. Castillo-Martin, A. Puzio-Kuter et al., "Targeting AKT/mTOR and ERK MAPK signaling inhibits hormone-refractory prostate cancer in a preclinical mouse model," Journal of Clinical Investigation, vol. 118, no. 9, pp. 3051-3064, 2008.

[43] Y. Fu, S. Wey, M. Wang et al., "Pten null prostate tumorigenesis and AKT activation are blocked by targeted knockout of ER chaperone GRP78/BiP in prostate epithelium," Proceedings of the National Academy of Sciences of the United States of America, vol. 105, no. 49, pp. 19444-19449, 2008.

[44] B. D. Manning, A. R. Tee, M. N. Logsdon, J. Blenis, and L. C. Cantley, "Identification of the tuberous sclerosis complex2 tumor suppressor gene product tuberin as a target of the phosphoinositide 3-kinase/Akt pathway," Molecular Cell, vol. 10, no. 1, pp. 151-162, 2002.

[45] K. Inoki, Y. Li, T. Zhu, J. Wu, and K. L. Guan, "TSC2 is phosphorylated and inhibited by Akt and suppresses mTOR signalling," Nature Cell Biology, vol. 4, no. 9, pp. 648-657, 2002. 


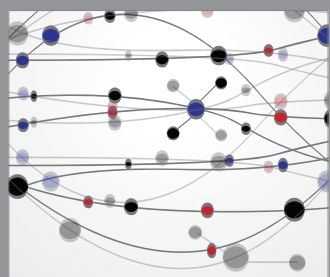

The Scientific World Journal
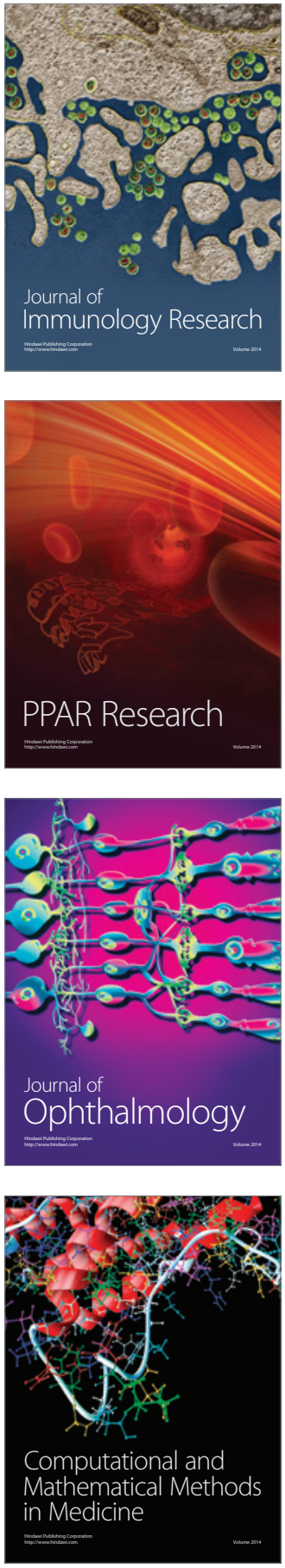

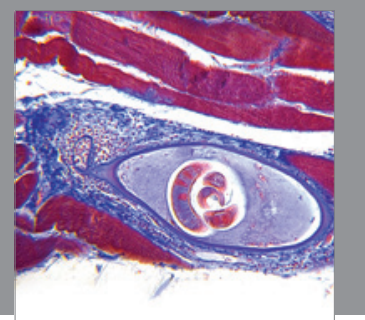

Gastroenterology

Research and Practice
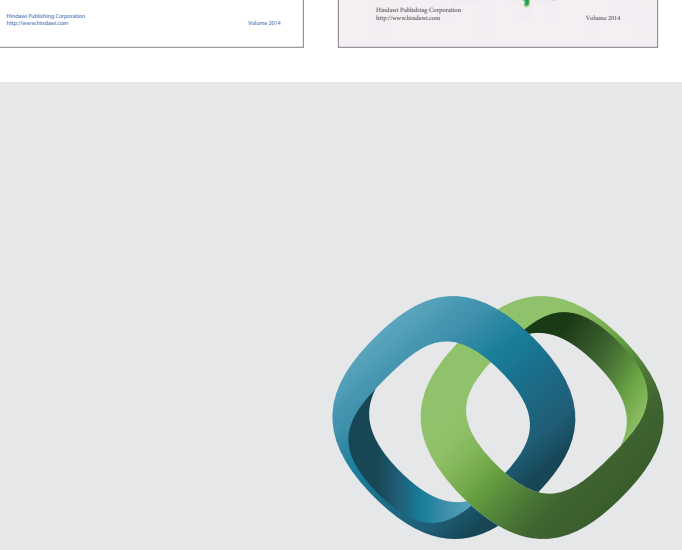

\section{Hindawi}

Submit your manuscripts at

http://www.hindawi.com
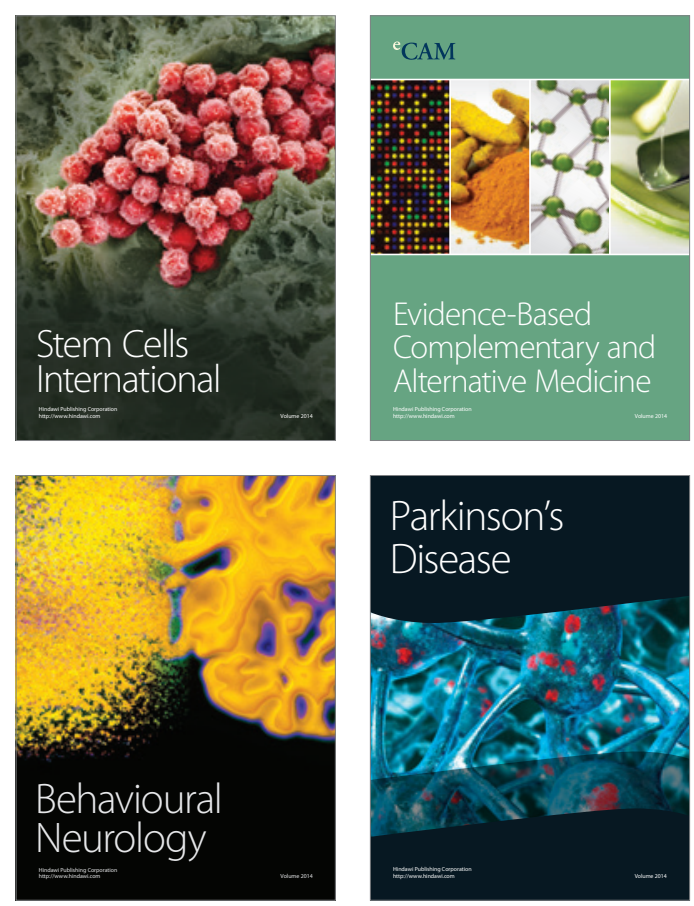

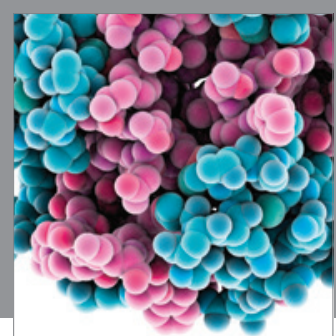

Journal of
Diabetes Research

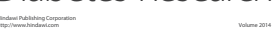

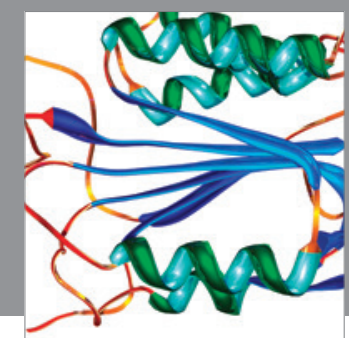

Disease Markers
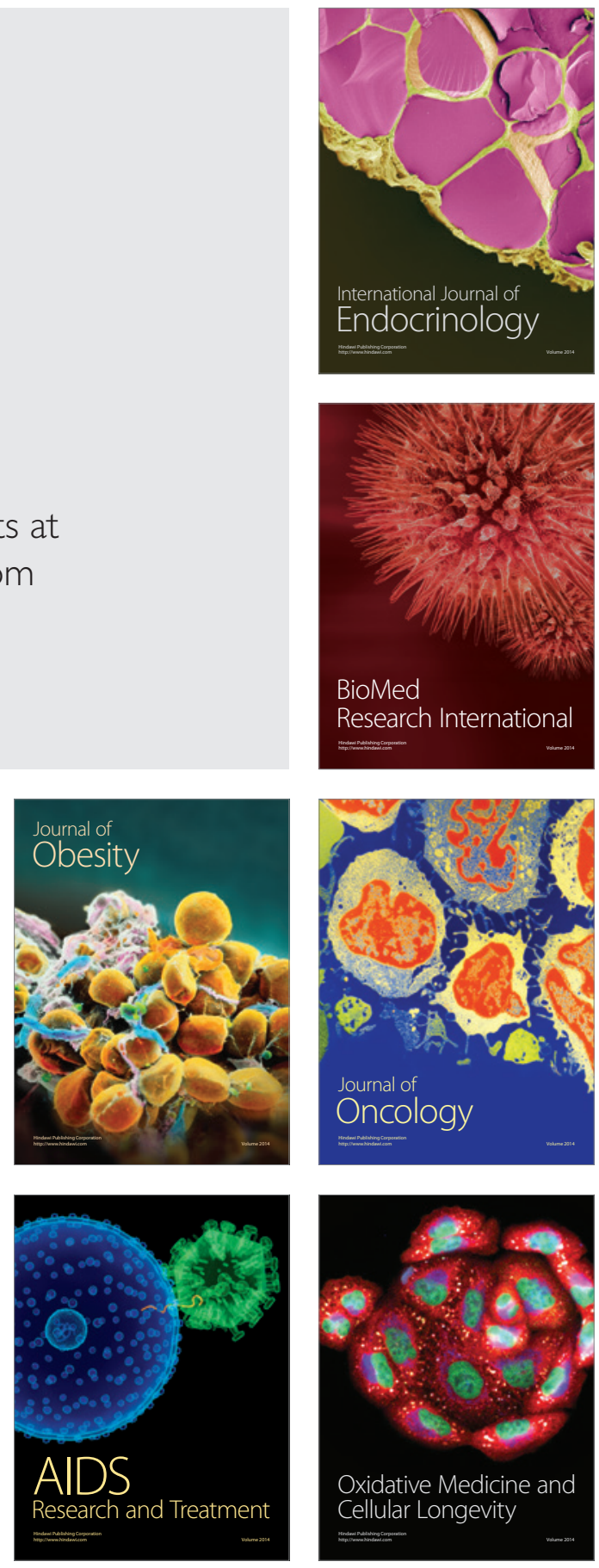